\title{
Effects of adult aging on utilization of temporal and semantic associations during free and serial recall
}

\author{
Julie D. Golomb, Jonathan E. Peelle, and Kelly M. Addis \\ Brandeis University, Waltham, Massachusetts \\ Michael J. KaHANa \\ University of Pennsylvania, Philadelphia, Pennsylvania \\ AND \\ ARTHUR WINGFIELD \\ Brandeis University, Waltham, Massachusetts
}

\begin{abstract}
Older adults show poorer performance than young adults at word list recall, especially for order information. In contrast with this temporal association deficit, older adults are generally adept at using preexisting semantic associations, when present, to aid recall. We compared the use of temporal and semantic associations in young and older adults' word list recall following both free recall and serial recall instructions. Decomposition of serial position curves confirmed that older adults showed weakened use of temporal context in recall in relation to young adults, a difference that was amplified in serial recall. Older adults' temporal associations were also less effective than young adults' when correlated with serial recall performance. The differential age decrement for serial versus free recall was accompanied by a persistent influence of latent semantic associations in the older adults, even when maladaptive for serial recall.
\end{abstract}

One of the hallmarks of healthy adult aging is a noticeable decline in memory performance. However, this cognitive deficit is highly selective; certain aspects of memory tend to be substantially impaired in older adults, whereas other aspects are remarkably well preserved. Episodic memory tasks, in which some temporal context must be associated with the content being held in memory, are particularly challenging for older adults (Burke \& Light, 1981; Kausler, 1994; Salthouse, 1991). Furthermore, within the spectrum of episodic memory tasks, older adults have the most difficulty with self-initiated recall of word lists, especially when the items are semantically unrelated (Burke \& Light, 1981; Craik, 1977; Kausler, 1994).

Results from various recall and recognition tasks have generated several theories about the causes of this episodic memory deficit in healthy older adults. Many of these have focused on fairly general explanations for this loss, such as reduced executive or working memory resources (see Light, 1991, for a review), and/or reduced processing speed (Salthouse, 1996). Others have cited an increased susceptibility to interference as a source of general cognitive decline (Zacks, Hasher, \& Li, 2000). These behavioral declines have been attributed to age-related neuronal loss, particularly in the prefrontal cortex, with its essential role for effective executive function (Bäckman,
Small, \& Wahlin, 2001; West, 1996). Consistent with this connection, functional imaging studies have shown the right prefrontal cortex to be more active during temporal order retrieval than during item retrieval in young adults, but not in older adults, establishing a more direct link between older adults' context memory deficits and frontal dysfunction (Cabeza, Anderson, Houle, Mangels, \& Nyberg, 2000).

Generalized theories, such as reduced resources or cognitive slowing, have been used to account for a wide range of memory deficits in adult aging, but most fail to explain the highly selective nature of these deficits. One of the most notable of these is a selective deficit in older adults' ability to make use of temporal associations between items in recall (Howard, Kahana, \& Wingfield, 2006; Kahana, Howard, Zaromb, \& Wingfield, 2002; NavehBenjamin, 2000). Naveh-Benjamin and colleagues found that older adults had increased difficulty remembering pairs of unrelated words in comparison with item memory for the words themselves, noting that this selective association deficit could not be accounted for by a simple decrease in attentional resources, which would predict a general memory impairment for both types of information (Naveh-Benjamin, Guez, \& Shulman, 2004; NavehBenjamin, Hussain, Guez, \& Bar-On, 2003).

A.Wingfield, wingfield@brandeis.edu 
To examine the use of associations in word list recall, Howard and Kahana (1999) described recall performance in terms of two elements: how participants initiate recall and how they make transitions between items once the recall has begun. During free recall tasks, young adults tend to begin recall with items occurring near the end of the list (Howard \& Kahana, 1999), as first noted by Deese and Kaufman (1957). When transitioning between items in recall, young adults display a characteristic pattern in which items that occurred together during presentation are more likely to be recalled together than are items that were presented farther apart; this is called the contiguity effect (Kahana, 1996). This effect is asymmetric, favoring forward associations over backward associations (Howard \& Kahana, 1999; Kahana, 1996). When these analyses were extended to older adults, the differentiation between these two processes was striking: The manner in which participants initiated recall was entirely unaffected by age, whereas older adults exhibited significantly decreased contiguity, illustrating a deficit in temporal organization (Howard et al., 2006; Kahana et al., 2002; Wingfield \& Kahana, 2002).

Studies of recall and aging often use free recall tasks, in which memorized items from a list can be recalled in any order (e.g., Wingfield \& Kahana, 2002). Although temporal organization of items may aid a participant during free recall, temporal organization is clearly more important for serial recall, in which task demands necessitate recalling list items in the order in which they were presented. It is important to note that the temporal organization we refer to here is based on the temporal order of list itemsthat is, the temporal context of an item with respect to another item (temporal context model; Howard \& Kahana, 2002a), not with respect to the absolute amount of time passed, which has little effect on serial recall (Lewandowsky, Brown, Wright, \& Nimmo, 2006). Because older adults appear to show weaker temporal organization during free recall, one might expect them to find the serial recall task particularly difficult. Interestingly, whereas a large number of studies have examined free recall and aging (see, e.g., Burke \& Light, 1981; Craik, 1977; Zacks et al., 2000), few have looked at serial recall and aging, and none have conducted a comparison of free and serial recall in older adults within a single experiment.

In the present experiment, we compared age-related changes in temporal associations during task conditions in which temporal organization was largely incidental (free recall) with those in which temporal organization was largely intentional (serial recall). It is possible that the decreased temporal organization seen in older adults is restricted to circumstances of incidental encoding, or that older adults use a different strategy — one that deemphasizes order information in order to maximize older adults' limited storage capacity for item information. By requiring both young and older adults to adopt a strategy that emphasizes the encoding of temporal order information, we can further probe the nature of this temporal association deficit in older adults.

In addition to examining temporal associations, we wished to determine the extent to which participants may also be making use of potential semantic associations among list items, as well as how the use of semantic information might vary with age and task demands. This question follows the classic distinction often made between episodic and semantic memory (Tulving, 1983). Extensive research has documented older adults' difficulties in episodic memory (Bäckman et al., 2001; Burke \& Light, 1981; Kausler, 1994; Salthouse, 1991), but semantic memory tends to be relatively spared (Burke \& Mackay, 1997). Using categorizable word lists, Wingfield, Lindfield, and Kahana (1998) demonstrated that both young and older adults tend to recall words clustered by semantic category (Bousfield, 1953), although older adults have more difficulty accessing the relevant categories. These findings led Wingfield and Kahana (2002) to formulate a hypothesis distinguishing between the ability to use preexperimental semantic associations, which is relatively spared in older adults, and the marked deficit in older adults' ability to use temporal associations, which must be created on line for any given word list.

We sought to test this hypothesis by using uncategorized word lists, in which temporal associations between list words would be more valuable during recall than any latent semantic associations that might happen to exist on a given list. During free recall tasks, we might expect participants to use a mixture of temporal and semantic associations. To succeed at serial recall, however, participants should shift to a temporally dominant strategy and make less use of potential semantic associations. We used two analyses to examine young and older adults' utilization of temporal context in list recall. One of these was the probability that an item from any given position in a presented list would be the first item to be recalled, as an indicator of participants' patterns of initiating recall. We refer to this measure as the probability of first recall (PFR; Laming, 1999). The second analysis examined the probability of an item being recalled as a function of its distance, either earlier or later in the presented list, from the item previously recalled. We refer to this as the lag-conditional response probability (lag-CRP), and we used this measure to assess temporal transitions made during recall and the strength of the contiguity effect (Howard \& Kahana, 1999; Kahana, 1996). In addition to obtaining these two measures, we also explored whether the strength of these temporal associations was correlated with overall recall accuracy for either task or age group. To probe semantic organization, we examined conditional response probability in terms of latent semantic associations between list items (semanticCRP; Howard \& Kahana, 2002b). These techniques are not new, but this is the first time these techniques have been used to compare associative abilities across age and task demands.

As a final factor, we also varied presentation rates for word list items. Our reason for testing different presentation rates was based on previous research showing that older adults are differentially impaired at recall, in comparison with young adults, when words are presented at faster rates (Kausler, 1994). Previous research has also shown, at least in young adults, that presentation rate selectively affects recall of pre-recency items (Glanzer \& 
Cunitz, 1966; Murdock, 1962), with which older adults tend to have particular difficulty (Craik, 1968, 1977). Our question was whether the effect of presentation rate would further interact with older adults' difficulty with order information during serial recall. Of interest was whether older adults might be differentially impaired in forming temporal associations at faster rates. From a theoretical point of view, increasing the presentation rate could impair the older adults in either of two ways: It might limit the amount of time for forming temporal context associations - an intentional process that should selectively affect serial recall —or it might lead to poor discrimination between the temporal context of two items - an incidental process that should affect both free and serial recall. We were primarily concerned with testing the first possibility.

Our goal was to decompose response tendencies during free and serial recall in order to determine the extent of temporal and semantic associations that may underlie word list recall. Such techniques might also elucidate the nature of the changes that accompany the well-established age deficits in word list recall (e.g., Kausler, 1994). We had three specific hypotheses in this study: (1) Older adults would show reduced ability to use temporal context, even in serial recall, in which recall order is essential to the task demands; (2) the ability to use temporal associations during serial recall would increase for older adults if the presentation rate of the list items were slowed; and (3) the age-related decline in effective use of temporal context in recall would be accompanied by an increased influence of semantic associations, even under serial recall instructions, in which this strategy would be maladaptive.

\section{METHOD}

\section{Participants}

There were 36 young adult participants and 36 older adult participants. The young group (16 men and 20 women) comprised university students $18-24$ years of age $(M=20.17, S D=1.65)$. They had a mean of 14.53 years of education at the time of testing $(S D=1.67)$ and a mean Wechsler Adult Intelligence Scale (WAIS-III; Wechsler, $1997)$ vocabulary score of $50.00(S D=6.80)$. Their mean forward and backward digit spans were 7.83 items $(S D=1.23)$ and 5.81 items $(S D=1.21)$, respectively.

The older group (16 men and 20 women) comprised communitydwelling adults $65-82$ years of age $(M=73.28, S D=4.27)$. They had a mean of 16.19 years of education $(S D=2.30)$ and a mean WAIS vocabulary score of $51.61(S D=5.74)$. Their mean forward and backward digit spans were 7.53 items $(S D=1.21)$ and 6.03 items $(S D=1.32)$, respectively.

Both groups were thus well-educated, with the older group having an average of 1.67 more years of formal education $[t(70)=3.52$, $p<.001]$. The two groups were statistically equivalent in tests of vocabulary $[t(70)=1.09$, n.s.], forward digit span $[t(70)=1.06$, n.s.], and backward digit $\operatorname{span}[t(70)=0.74$, n.s. $]$. All participants were native English speakers and reported themselves to be in good health. They were compensated with a small monetary sum for their participation.

\section{Stimuli}

Stimuli were 846 two-syllable nouns chosen from a restricted range of ratings for concreteness $(M=457.57, S D=114.95)$, familiarity $(M=528.33, S D=53.45)$, imagery $(M=482.36, S D=99.27)$, and Kučera-Francis frequency $(M=58.44, S D=64.27$; Kučera \& Fran- cis, 1967). Each participant received a total of 80 lists of 10 words each, selected at random and without replacement from the noun pool.

\section{Procedure}

The experiment was conducted in two separate sessions of approximately $40 \mathrm{~min}$ each. For half of the participants, their first session was a free recall task, and their second session was a serial recall task. The other participants performed the tasks in the reverse order. The sessions occurred at least 1 week apart to minimize possible carryover in strategy from the previous session's tasks.

At the start of the task, participants were given instructions for either free or serial recall, followed by 4 practice trials. The practice trials were immediately followed by 36 test trials. Participants were cued to press the spacebar to begin each trial. A tone signaled the beginning of presentation. Words were presented one at a time in capital letters in the center of the computer screen for $1,000 \mathrm{msec}$, followed by a blank interstimulus interval (ISI). ISIs were $800 \mathrm{msec}, 1,200 \mathrm{msec}$, or 2,400 msec. Within a given list, all ISIs were the same, and trials were blocked according to ISI. Each session consisted of one block of 12 trials for each of the three presentation rates. The order of these blocks was counterbalanced across participants, and each participant received the same order for both of their sessions. Participants were informed at the beginning of each block whether they would be receiving the "fast," "medium," or "slow" presentation rate.

As each word appeared on the screen, participants were instructed to say the word aloud. This ensured that all participants attended to and perceptually processed each word in the list. After the final word was presented, three asterisks appeared on the screen, accompanied by a tone. This was the signal to begin recall. For free recall, participants were instructed to recall words from the list in any order; for serial recall, they were instructed to attempt to recall the words in the order they were presented. The serial recall instructions explicitly encouraged participants to begin recall with the first list item; however, because of the supraspan list length, if they were unable to retrieve the first item, they were instructed to begin recall with the earliest item they could remember. Participants were not required to make exactly 10 responses, nor were they instructed to say "skip" or "pass" during serial recall.

Participants' recall responses were recorded on the computer, using a microphone. Participants were instructed to speak loudly and clearly and to avoid repetitions and extraneous noises. They were given up to $1 \mathrm{~min}$ to recall as many words from the list as possible, although they could press the spacebar at any point to indicate they were finished recalling. Participants were then given the option either to take a break or to continue on to the next trial.

\section{Response Scoring}

For free recall tasks, item scoring was used, in which recalled words were scored as being correct if they had appeared in the most recent list, regardless of their position in the order of response. Serial recall tasks used relative-order scoring, in which a recalled word was considered to be correct only if it appeared later in the list than the previously recalled item (Drewnowski \& Murdock, 1980). The first item recalled was always scored as being correct. Relative-order scoring has been used as a standard measure in many previous studies of this nature (e.g., Addis \& Kahana, 2004; Drewnowski \& Murdock, 1980; Klein, Addis, \& Kahana, 2005; Nyberg et al., 2003; Reeves \& Sperling, 1986).

Although relative-order scoring has its drawbacks, such as giving an automatic boost to later list items (the final list item is scored as being correct regardless of its recall position), it has nonetheless been demonstrated to be a more appropriate measure of performance in serial recall tasks, avoiding many of the problems inherent in using item scoring or strict-order scoring for word lists of this length (see Addis \& Kahana, 2004; Klein et al., 2005). For example, strict-order scoring can be unreliable for oral recall of supraspan lists, putting later items at an extreme disadvantage - that is, the final list item could only be scored as being correct if exactly nine responses preceded it. Because it can be quite cumbersome to keep count of the number of "skips" or "passes" in addition to the list 

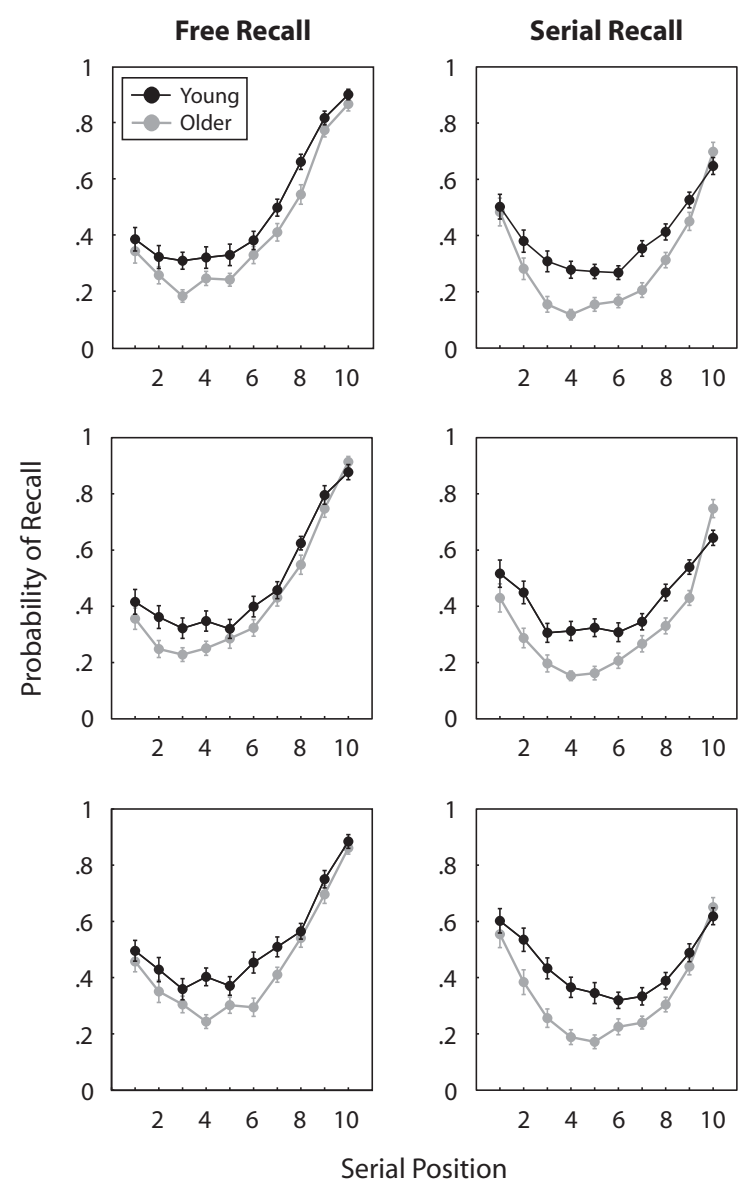

Figure 1. Serial position curves. The probability of correctly recalling a word on the basis of serial position is shown for free recall tasks (left panels) and serial recall tasks (right panels). Black lines represent values for young adults; gray lines represent those for older adults. Top panels: Fast presentation rate $(800-\mathrm{msec}$ ISI). Middle panels: Medium presentation rate (1,200-msec ISI). Bottom panels: Slow presentation rate $(2,400-m s e c$ ISI). Error bars represent one standard error. Absence of error bars indicates that they were too small to plot.

words under these conditions, we did not instruct participants to use strict positional recall in this task; thus, strict-order scoring would be unsuitable here, critically underestimating recall performance. Item scoring, on the other hand, overestimates performance on the serial recall task, since order information is not taken into account. However, as an additional means of comparison, we analyzed our serial recall data using item scoring as well as relative-position scoring; where the results differed, we report statistics for both scoring methods. When not otherwise indicated, serial recall results are based on relative-order scoring. For both scoring methods, plural versions of list items were considered correct.

\section{RESULTS}

\section{Serial Position Effects}

Figure 1 displays the serial position curves for young and older adults performing free recall (left panels) and serial recall (right panels) tasks at each of the three presentation rates, represented as the probability of correctly recalling an item as a function of its serial position in the presented list. The free recall plots all show the traditional serial position curves with large recency and smaller primacy effects, and the serial recall curves display a relative increase in primacy and a decrease in recency. In relation to those of the young adults, the older adults' performance deficits appear primarily in the early to middle serial positions, with performance during the recency portion largely intact.

To compare the shapes of these serial position curves, the data were submitted to a 3 (rate: fast, medium, slow) $\times 2$ (task: free recall, serial recall) $\times 10$ (serial position: 1-10) $\times 2$ (age: young, older) mixed design ANOVA, with rate, task, and serial position as withinparticipants variables. The young group correctly recalled a greater number of words per list than did the older group, as supported by a significant main effect of age $\left[F(1,70)=15.78, M S_{\mathrm{e}}=.47, p<.001\right]$. The main effects of task $\left[F(1,70)=211.23, M S_{\mathrm{e}}=.05, p<.001\right]$ and rate $\left[F(2,140)=24.03, M S_{\mathrm{e}}=.02, p<.001\right]$ were also significant, confirming that serial recall tasks were more difficult than free recall tasks, and that slower presentation rates resulted in improved performance on both tasks. The task $\times$ age interaction was significant $[F(1,70)=$ $\left.4.48, M S_{\mathrm{e}}=.05, p<.05\right]$, indicating that older adults performed differentially poorer on the serial recall tasks. When item scoring was used for both tasks, the task $X$ age interaction was no longer significant $(F<1)$, confirming that the older adults' serial recall deficit was due to decreased use of order information; item information was relatively preserved. Neither the rate $\times$ age $(F<1)$, task $\times$ rate $\left[F(2,140)=1.88, M S_{\mathrm{e}}=.01\right]$ nor task $\times$ rate $\times$ age $(F<1)$ interactions were significant.

Because the factor of serial position violated the sphericity assumption, epsilon values for Greenhouse-Geisser corrections are provided for all tests involving this factor. There was a significant main effect of serial position $\left[F(9,630)=142.79, M S_{\text {e }}=.09, p<.001, \varepsilon=.24\right]$, indicating that the probability of correct recall varied as a function of serial position. The task $\times$ serial position interaction was significant $\left[F(9,630)=56.39, M S_{\mathrm{e}}=.03\right.$, $p<.001, \varepsilon=.60]$, confirming that the shapes of these curves differed according to whether participants were performing free or serial recall tasks. Presentation rate also affected the shapes of the curves, with slower presentation rates resulting in stronger primacy effects, supported by a significant rate $\times$ serial position interaction $\left[F(18,1260)=8.29, M S_{\mathrm{e}}=.02, p<.001, \varepsilon=.69\right]$. The three-way rate $\times$ task $\times$ serial position interaction was not significant $(F<1)$, nor were any of these interactions further moderated by age, with no significant interactions of task $\times$ serial position $\times$ age $\left[F(9,630)=1.60, M S_{\mathrm{e}}=\right.$ $.03, \varepsilon=.60]$, rate $\times$ serial position $\times$ age $[F(18,1260)=$ $\left.1.39, M S_{\mathrm{e}}=.02, \varepsilon=.69\right]$, or rate $\times$ task $\times$ serial position $\times$ age $(F<1)$. However, there was a marginally significant serial position $\times$ age interaction $[F(9,630)=$ $\left.2.77, M S_{\mathrm{e}}=.09, p<.065, \varepsilon=.24\right]$, supporting the trend that for both free and serial recall tasks, older adults had more difficulty with certain serial positions - particularly those falling in the middle of the list. 
Free Recall
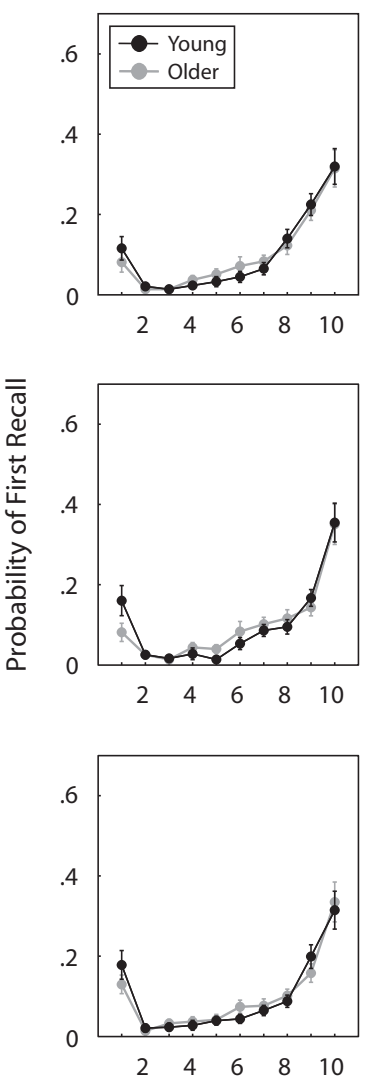

Serial Recall
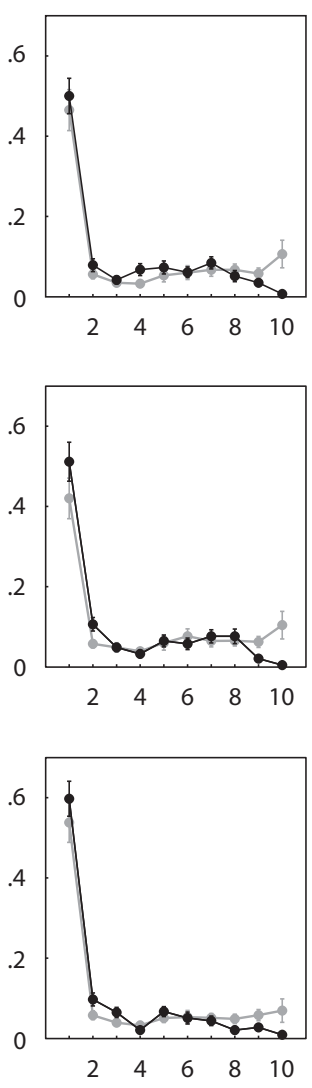

Serial Position

Figure 2. Probability of first recall. The probability of initiating recall based on serial position is shown for free recall tasks (left panels) and serial recall tasks (right panels). Black lines represent values for young adults; gray lines represent those for older adults. Top panels: Fast presentation rate (800-msec ISI). Middle panels: Medium presentation rate $(1,200-m s e c$ ISI). Bottom panels: Slow presentation rate (2,400-msec ISI). Error bars represent one standard error.Absence of error bars indicates that they were too small to plot.

\section{Probability of First Recall (PFR)}

To examine potential age differences in how young and older adults initiated recall, we plotted the probability that an item from any particular serial position would be the first item recalled. This is represented as the PFR, as a function of the item's serial position in the presented list. These PFR plots are shown in Figure 2 for free recall (left panels) and serial recall (right panels) for each of the three presentation rates for the two age groups. During free recall, both young and older adults tended to begin recall with items from the final few positions in a list, with a very low probability of beginning recall with the first word and almost zero probability for words falling in the middle of a list, a pattern well established in previous studies (Deese \& Kaufman, 1957; Howard \& Kahana, 1999). In contrast, when participants were instructed to recall the word list in order in the serial recall condition, both age groups dem- onstrated their capability to override the tendency to recall recent words first, typically (and appropriately) beginning recall with the first item in the list.

The PFR data shown in Figure 2 were submitted to a 3 (rate: fast, medium, slow) $\times 2$ (task: free recall, serial recall) $\times 10$ (serial position: $1-10) \times 2$ (age: young, older $)$ mixed design ANOVA, with rate, task, and serial position as within-participants variables. The main effects of task $\left[F(1,70)=11.95, M S_{\mathrm{e}}<.01, p<.001\right]$ and serial position $\left[F(9,630)=64.41, M S_{\mathrm{e}}=.05, p<.001, \varepsilon=\right.$ $.28]$ were significant, as was the task $\times$ serial position interaction $\left[F(9,630)=101.57, M S_{\mathrm{e}}=.03, p<.001\right.$, $\varepsilon=.36]$, confirming the relative differences in strengths of recency and primacy effects in initiating recall in the free and serial recall tasks.

The rate $\times$ serial position interaction was significant $\left[F(18,1260)=5.44, M S_{\mathrm{e}}<.01, p<.001, \varepsilon=.58\right]$, supporting the observation that as presentation rate slowed, the probability of initiating recall with the first word increased. This interaction was further modified by a significant rate $\times$ task $\times$ serial position interaction $\left[F(18,1260)=1.90, M S_{\mathrm{e}}<.01, p<.05, \varepsilon=.56\right]$, confirming the appearance that this effect was more pronounced in the serial recall than in the free recall task. The rate $\times$ task interaction was marginally significant $\left[F(2,140)=2.94, M S_{\mathrm{e}}<.01, p=.056\right]$, and the main effect of rate approached significance as well $[F(2,140)=$ 2.43, $\left.M S_{\mathrm{e}}<.01, p=.092\right]$.

Overall, the PFR was not affected by age. The main effect of age was not significant $\left[F(1,70)=1.08, M S_{\mathrm{e}}<\right.$ $.01]$, nor did age moderate any other main effects or interactions [rate $\times$ age, $F<1$; task $\times$ age, $F(1,70)=$ $1.83, M S_{\mathrm{e}}<.01$; serial position $\times$ age, $F(9,630)=1.42$, $M S_{\mathrm{e}}=.05, \varepsilon=.28$; rate $\times$ task $\times$ age, $F<1$; rate $\times$ serial position $\times$ age, $F<1$; task $\times$ serial position $\times$ age, $F(9,630)=1.38, M S_{\mathrm{e}}=.03, \varepsilon=.36$; rate $\times$ task $\times$ serial position $\times$ age, $F<1$ ].

\section{Conditional Response Probability (CRP) As a Function of Lag}

The PFR curves illustrate how participants initiate recall; it is also valuable to determine how participants make transitions between items being recalled. Kahana (1996) first looked at the relationship between two consecutive recalls and the number of items that separated them (lag) in the presented list. This information is used to calculate the CRP as a function of lag (lag-CRP). The lag-CRP measures the probability that a subsequent recall will reflect a transition of a given lag, conditionalized on that lag's availability. For example, if items $a, b, c$, and $d$ are presented in that order, $d$ is at a lag of +1 from $c$, and $a$ is at a lag of -2 from $c$. If item $c$ is recalled, a subsequent recall from that list could reflect possible transitions of lag $-2,-1$, or +1 . If the subsequent recall is item $b$, that would increase the response probability at lag -1 , decrease the probability at lags -2 and +1 , and leave all other lags unaffected.

The lag-CRP is shown in Figure 3 for the young and older adults for free and serial recall at each of the tested presentation rates. Since there was very little power at ex- 


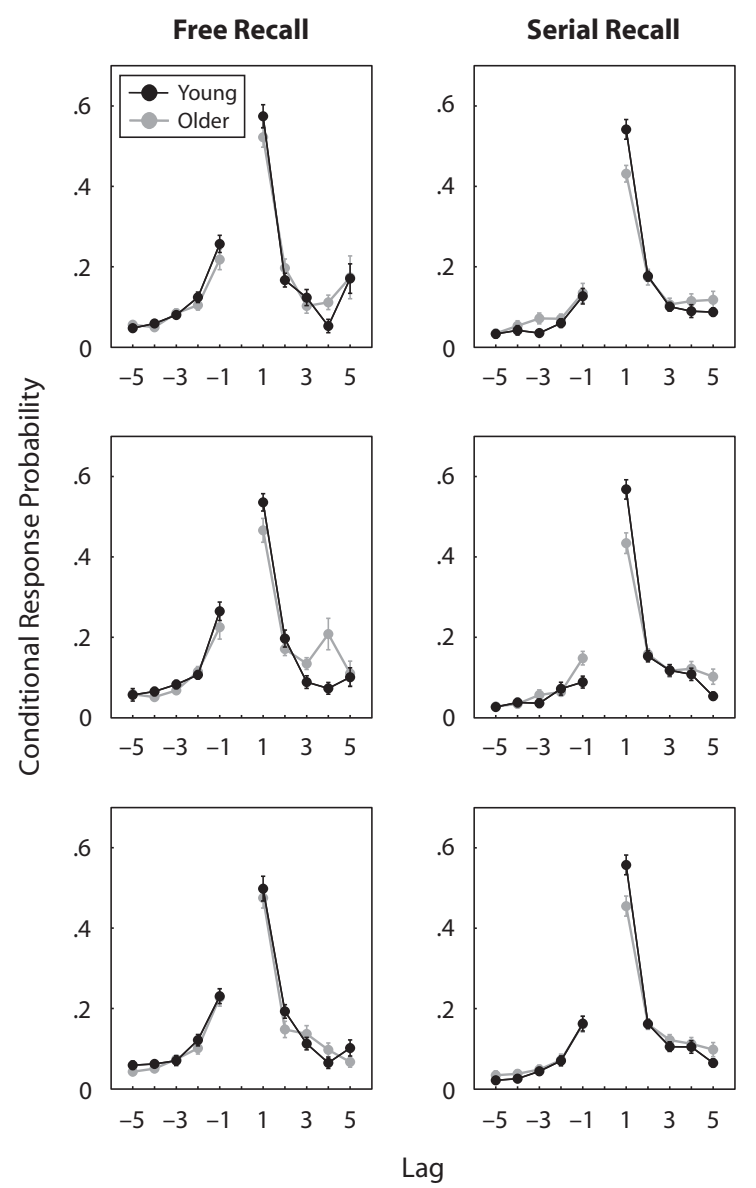

Figure 3. Conditional response probabilities. The conditional response probability, as a function of temporal lag (lag-CRP), is shown for free recall tasks (left panels) and serial recall tasks (right panels). Black lines represent values for young adults; gray lines represent those for older adults. Top panels: Fast presentation rate (800-msec ISI). Middle panels: Medium presentation rate (1,200-msec ISI). Bottom panels: Slow presentation rate (2,400-msec ISI). Error bars represent one standard error. Absence of error bars indicates that they were too small to plot.

tremely remote lags, analyses were restricted to the range of lag -5 to lag +5 . For free recall, Figure 3 shows that both young and older adults demonstrated a typical pattern of forward contiguity (Howard \& Kahana, 1999; Kahana, 1996; Kahana et al., 2002). Items that occurred together during presentation (adjacent items, or short lags) were more likely to be recalled together than items that were presented farther apart (remote items, or long lags), and forward associations were favored over backward associations. The pattern was similar for the serial recall tasks, although there appeared to be a more pronounced forward asymmetry than in the free recall tasks, reflecting the increased use of order information during serial recall.

To quantify these observations, we categorized the lag positions according to distance (adjacent or remote) and direction (forward or backward). Lags +1 and -1 were considered adjacent positions, and lags +3 to +5 and -3 to -5 were considered remote positions. To test the contiguity effect, paired samples $t$ tests were conducted, comparing conditional response probabilities at adjacent and remote lags for each condition. Adjacent lags had significantly higher recall probabilities than did remote lags in both forward and backward directions for all rates, both tasks, and age groups (all $p \mathrm{~s}<.001$ ), confirming the robust bias for jointly recalling items that were presented together.

To further probe this effect, we took the difference in response probability (adjacent - remote) as a measure of contiguity for each condition. A 3 (rate: fast, medium, slow) $\times 2$ (task: free recall, serial recall) $\times 2$ (lag direction: backward, forward) $\times 2$ (age: young, older) mixed design ANOVA was conducted on the contiguity difference scores, with rate, task, and lag direction as withinparticipants variables. There was a significant main effect of age $\left[F(1,70)=12.52, M S_{\mathrm{\iota}}=.05, p<.001\right]$, indicating that older adults showed a weaker contiguity effect than did younger adults, a result analogous to overall age-related differences in recall accuracy (Kahana et al., 2002). The forward asymmetry effect was confirmed by a significant main effect of lag direction $\left[F(1,70)=246.31, M S_{\mathrm{e}}=\right.$ $.06, p<.001]$, demonstrating that participants were more likely to make recall transitions to an adjacent item in the forward direction than in the backward direction. Most important, however, was a significant lag direction $\times$ age interaction $\left[F(1,70)=8.92, M S_{\mathrm{e}}=.06, p<.005\right]$, indicating that older adults used less of this order information in recall than did young adults.

There was also a significant main effect of task $\left[F(1,70)=11.90, M S_{\mathrm{e}}=.03, p<.001\right]$, revealing that participants exhibited more contiguity in free recall than in serial recall. Although this may seem counterintuitive, it is important to note that this main effect did not differentiate between contiguous recall transitions made in the forward and backward directions. Indeed, a significant task $\times$ lag direction interaction $\left[F(1,70)=5.70, M S_{\mathrm{e}}=\right.$ $.05, p<.05]$ supported this distinction; the decreased overall contiguity in serial recall in comparison with that in free recall appeared to be driven by a decrease in the probability of contiguous recall in the backward direction. That is, participants exhibited stronger forward asymmetry in the serial recall task than in the free recall task, an expected finding, given the emphasis on order information in serial recall. Neither the task $\times$ age $(F<1)$, nor the task $\times$ lag direction $\times$ age $\left[F(1,70)=2.36, M S_{\mathrm{e}}=.05\right]$ interactions were significant.

Presentation rate did not influence overall measures of temporal contiguity, as evidenced by the lack of a significant main effect of rate $(F<1)$. There was also no significant rate $\times$ age interaction $(F<1)$. It is interesting, however, that there was a significant rate $\times$ task interaction $\left[F(2,140)=4.15, M S_{\mathrm{e}}=.01, p<.05\right]$, revealing a pattern in which slowing the presentation rate resulted in stronger temporal contiguity for serial recall tasks and weaker temporal contiguity for free recall tasks. The rate $\times$ task $\times$ age interaction approached significance $\left[F(2,140)=2.56, M S_{\mathrm{e}}=.01, p=.081\right]$, suggesting that age may further moderate these response patterns. 
More specifically, as presentation rate was slowed, young adults exhibited a strong decrease in free recall contiguity and a weak increase in serial recall contiguity. For older adults, on the other hand, slower presentation rates tended to produce a weaker change in free recall contiguity, but a stronger increase in serial recall contiguity. Thus, in terms of making correct recall transitions during serial recall tasks, older adults may have benefited more from the longer presentation times than did young adults. The rate $\times$ lag direction $(F<1)$, rate $\times$ lag direction $\times$ age $(F<1)$, rate $\times$ task $\times$ lag direction $[F(2,140)=$ $\left.1.68, M S_{\mathrm{e}}=.02\right]$, and rate $\times$ task $\times$ lag direction $\times$ age $(F<1)$ interactions were not significant.

\section{Forward Contiguity and Recall Accuracy}

Because most recall transitions were concentrated at the lag +1 position, we asked whether the strength of this forward contiguity effect might be directly related to overall performance. For each word list, we took the probability of a lag +1 response as a measure of forward contiguity and the overall percent correct as a measure of accuracy, and we used these paired values to calculate regression slopes on a participantwise basis. Table 1 displays the average regression slopes for each condition and age group, as well as for the collapsed data. Positive slopes indicate that higher probabilities of correct lag +1 transitions were more likely to be associated with higher overall levels of accuracy.

To compare the relationship between forward contiguity and recall accuracy across conditions, we submitted the regression slopes to a 3 (rate: fast, medium, slow) $\times$ 2 (task: free recall, serial recall) $\times 2$ (age: young, older) mixed design ANOVA, with rate and task as withinparticipants variables. There was a significant main effect of age $\left[F(1,70)=4.17, M S_{\mathrm{e}}=3.59, p<.05\right]$, suggesting that young adults made better use of order information during recall than did older adults. The main effect of task was also significant $\left[F(1,70)=64.40, M S_{\mathrm{e}}=2.10, p<\right.$ $.001]$, implying that forward contiguity was more integral to successful performance during serial recall than during free recall, a result consistent with the role order that information plays in the two tasks. This main effect of task was modulated by a significant task $\times$ age interaction $\left[F(1,70)=5.65, M S_{\mathrm{e}}=2.10, p<.05\right]$. Post hoc $t$ tests elaborated that the regression slopes did not significantly differ between young and older adults during free recall $[t(35)=0.29]$, but that the age difference was significant during serial recall $[t(35)=2.91, p<.01]$, further highlighting the decreased use of order information during serial recall with age.
When item scoring was used to obtain the accuracy measure instead of relative-order scoring, the main effect of task was weaker, but still significant $[F(1,70)=$ $\left.4.49, M S_{\mathrm{e}}=2.08, p<.05\right]$, whereas the main effect of age and the task $\times$ age interaction were no longer significant $\left[F(1,70)=2.34, M S_{\mathrm{e}}=2.89\right.$, and $F(1,70)=$ $2.27, M S_{\mathrm{e}}=2.08$, respectively], confirming that the relationship between contiguity and recall accuracy is indeed due to use of order information during recall. Regardless of scoring method, the main effect of rate was not significant $(F<1)$, nor were any interactions involving this factor (rate $\times$ age, $F<1$; rate $\times$ task, $F<1$; rate $\times$ task $\times$ age, $F<1$ ).

\section{Latent Semantic Analysis}

Although the word lists used in this experiment were selected randomly, with no intentional semantic relatedness, past literature has suggested that individuals will often make use of subtle semantic associations between list items in word list recall (Howard \& Kahana, 2002b). Utilization of these semantic associations should manifest as an increase in the probability of recalling words together that happen to be more semantically related. In order to investigate the role that semantic information might play in free and serial recall tasks, we used latent semantic analysis (LSA; Landauer \& Dumais, 1997) to derive a measure of semantic similarity between all possible word pairs that could appear together in our word lists. This measure of lexical co-occurrence has been used in previous studies to assess the degree to which semantic similarity can influence recall transitions by calculating conditional response probability as a function of LSA similarity (semanticCRP; Howard \& Kahana, 2002b).

LSA is based on the assumption that words similar in meaning tend to appear together in written text: Any word can be represented as a vector in a high-dimensional semantic space on the basis of its probability of cooccurrence with other words. The cosine of the angle $\theta$ between two vectors (or words) reflects their semantic similarity: Values close to 1 indicate high levels of semantic similarity between a pair of words, whereas values close to 0 indicate very little similarity between the pair. To calculate the semantic-CRP, we first obtained the $\cos (\theta)$ values for each possible pair of words drawn from our 846-word list. These values were then divided into seven logarithmically spaced bins spanning the range of $\cos (\theta)$ values. Because the distribution of $\cos (\theta)$ values was highly skewed-approximately $80 \%$ of word pairs found in this study had $\cos (\theta)$ values between 0 and

Table 1

Relationship Between Lag + 1 Recall Probability and Recall Accuracy: Regression Slopes

\begin{tabular}{|c|c|c|c|c|c|c|}
\hline \multirow[b]{2}{*}{ Presentation Rate } & \multicolumn{3}{|c|}{ Free Recall } & \multicolumn{3}{|c|}{ Serial Recall } \\
\hline & Young & Older & Overall & Young & Older & Overall \\
\hline Fast (800 msec) & 0.019 & -0.202 & -0.091 & 1.353 & 0.721 & 1.037 \\
\hline Medium $(1,200 \mathrm{msec})$ & -0.007 & 0.088 & 0.041 & 1.383 & 0.879 & 1.131 \\
\hline Slow $(2,400 \mathrm{msec})$ & 0.014 & 0.018 & 0.016 & 1.644 & 0.668 & 1.156 \\
\hline Overall & 0.077 & 0.012 & 0.044 & 1.442 & 0.774 & 1.108 \\
\hline
\end{tabular}




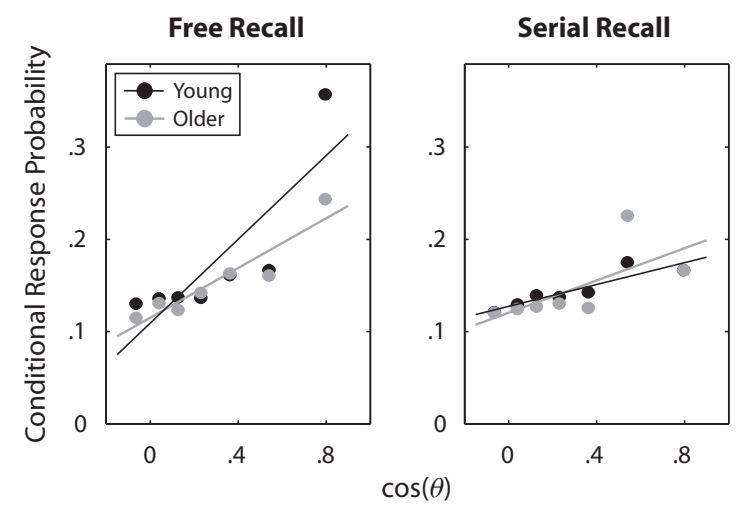

Figure 4. Semantic associations. Conditional response probability, as a function of latent semantic similarity (LSA-CRP), is shown for free recall tasks (left panel) and serial recall tasks (right panel). Black data points and black regression line represent values for young adults; gray data points and gray regression line represent values for older adults. Data are collapsed across rate. Explanation of LSA $\cos (\theta)$ values is given in the text.

0.2 - the logarithmically spaced bins allowed us to sample from the more extreme values while still maintaining statistical reliability. For each bin, we calculated the conditional probability of successfully recalling words whose semantic similarity fell within that bin.

The resulting semantic-CRPs are shown in Figure 4 for each age group and recall task. (Data were collapsed across presentation rate to maximize statistical power.) The mean $\cos (\theta)$ of the bin is plotted along the $x$-axis, and the probability of recall is plotted along the $y$-axis. Thus, each data point reflects the probability of successively recalling two words whose LSA $\cos (\theta)$ falls within a given bin. Best-fit linear regression lines for each data set are shown separately for the young and older adults.

As evidenced by the positive slopes of the semantic-CRP functions, semantic information influenced recall for both young and older adults during free and serial recall tasks. However, the extent to which semantic information was used differed on the basis of the task and participant group. In order to compare the semantic association effects, we performed two-tailed $t$ tests on the linear regression slopes for each age group and task using the following formula:

$$
t=\frac{b_{1}-b_{2}}{\sqrt{s_{b_{1}}^{2}+s_{b_{2}}^{2}}}, d f=n_{1}+n_{2}-4,
$$

where $b_{1}$ and $b_{2}$ are the slopes being compared, $s_{b_{1}}$ and $s_{b_{2}}$ are the standard errors of the slopes, and $n_{1}$ and $n_{2}$ are the numbers of bins.

The young adults relied significantly less on semantic information during serial recall than during free recall $[t(10)=2.50, p<.05]$, presumably to accommodate an increased dependence on temporal information. Older adults, on the other hand, continued to use similar levels of semantic information in serial recall $[t(10)=1.00$, n.s.]. This persistent use of semantic information, which is maladaptive for a serial recall task, may reflect an ad- ditional source of older adults' increased difficulty with serial recall tasks.

\section{DISCUSSION}

The serial position curves in this experiment took the traditional shapes for free and serial recall, with free recall showing a very strong recency effect and a weaker primacy effect (Murdock, 1962), whereas serial recall showed a weaker recency and stronger primacy effect (Deese, 1957; Jahnke, 1965). The effects of adult aging were most apparent on recall of items that appeared in the middle of the word lists, with the young and older adults showing similar accuracy levels for the first word and last few words. Consistent with the existing literature, our results confirmed that older adults perform worse than young adults in both free and serial recall.

For both age groups, serial recall was shown to be more difficult than free recall, resulting in fewer correct responses. Serial recall was expected to be the more difficult task, by virtue of its more demanding requirement of memory for order information as well as item information (Jahnke, 1965; Klein et al., 2005). The present experiment added to this basic finding by directly showing that older adults' decreased performance in recall tasks is differentially impaired during serial recall, in comparison with relatively smaller impairments in free recall. This outcome would be expected if adult aging resulted in diminished effectiveness in utilizing temporal context information in recall (see Kausler, 1994, for a review).

It could be argued that the older adults' poorer performance on serial recall tasks might be due to their forgetting the first few words of the list: If participants began their recall with a word appearing late in the list, this would limit the number of possible words that could be subsequently recalled in order; thus, overall performance would suffer. The analysis of the PFR data argues convincingly against this possibility, however, because the older participants were just as likely to begin their recall at the beginning of the list as were the young participants. A second possibility, strongly supported by the present data, is that the older adults' deficits in order memory were due to poorer temporal organization of the word lists (Howard et al., 2006; Kahana et al., 2002; Naveh-Benjamin, 2000). Age differences in the contiguity effect support this interpretation, with older adults showing much weaker forward contiguity (i.e., lag +1 recall probability), implying that they were less likely than the young adults to maintain temporal order information during recall.

In a free recall study, Kahana et al. (2002) compared young and older adults in terms of how they initiated recall and how they made transitions among neighboring items. The authors found that both young and older adults initiated recall in the same manner, but that older adults' recall transitions were less influenced by temporal proximity of the study items than were the young adults'. In the present study, we demonstrated that these age differences were further amplified in serial recall in comparison with free recall. To accommodate the serial order instructions, young adults appeared to shift strategy for the serial re- 
call task (in comparison with free recall) by exhibiting a stronger forward asymmetry in their contiguity effect in addition to initiating recall with earlier items. The older adults also successfully altered their strategy by appropriately initiating recall with earlier list items; but unlike the young adults, they did not show stronger asymmetry. These results suggest that the older adults were attempting to follow serial recall instructions, but that they were limited in their ability to temporally organize the word lists. This lack of temporal organization is consistent with recent work that demonstrates older adults' deficits in maintaining order information (e.g., Cabeza et al., 2000) and in creating associations between units of information (Naveh-Benjamin, 2000; Naveh-Benjamin et al., 2004; Naveh-Benjamin et al., 2003).

Our present results suggest that this temporal association deficit is not simply a lack of incidental encoding of temporal context, as might occur during free recall tasks. The fact that this deficit was even more pronounced in serial recall, in which order information was to be intentionally encoded, demonstrates that memory for temporal associations or context is truly impaired in older adults. This conclusion is consistent with that of Naveh-Benjamin (2000), who described an analogous deficit using a paired associates task. When young adults were instructed to focus on remembering associations between word pairs, they performed better on tests of associative memory than when they were instructed to focus on remembering item information only. However, older adults displayed no such benefit when the associations were to be intentionally - as opposed to simply incidentally — encoded (Naveh-Benjamin, 2000). This pattern was amplified in our present results, which also revealed age differences in the effectiveness of these temporal associations. In comparison with that of the older adults, young adults' recall accuracy was significantly more correlated with the conditional response probability at the +1 lag, demonstrating that the more likely a young adult was to maintain a word's temporal context during recall, the more correct words he or she was likely to recall from that list. This correlation was amplified during serial recall, although to a much lesser extent for the older adults. Thus, even when older adults were able to make recall transitions reflecting retention of temporal context, they tended to be less able to make use of this temporal information to support their recall.

In the presence of this temporal association deficit, the older adults were seen to rely more heavily on semantic organization to guide list retrieval, even when this would be maladaptive, as in the case of serial recall. This tendency was revealed in the comparison between the lag-CRP and the semantic-CRP. During free recall, both young and older adults used semantic information in addition to temporal information, confirming and extending the Howard and Kahana (2002b) results. Although the older adults exhibited slightly weaker associations of both semantic and temporal information, the age differences were modest, which was consistent with the relatively smaller age difference in free recall accuracy. In the serial recall task, on the other hand, young adults appeared to shift strategy from a mixed temporal/semantic organization to a pre- dominantly temporal organization. That is, in comparison with the free recall task, young adults exhibited stronger temporal associations (stronger forward contiguity effect in lag-CRP) and weaker semantic associations (weaker correlation in semantic-CRP) during serial recall. The older adults, however, demonstrated a strikingly different pattern than that of the young adults during serial recall: The older adults did not shift to a predominantly temporal organization, instead persisting in their reliance on semantic associations. Because task order was counterbalanced, this persistence cannot be attributed to difficulties adopting new task instructions.

The relative sparing of semantic versus temporal abilities in older adulthood is consistent with previous reports (Burke \& Mackay, 1997; Wingfield \& Kahana, 2002; Wingfield et al., 1998). Thus, older adults' stronger use of semantic associations during serial recall could be either a cause or an effect of their weaker reliance on temporal associations. On one hand, older adults may be less able to override the more natural tendency to associate items that are semantically related. To the extent that older adults experience reduced frontal lobe function (Bäckman et al., 2001; West, 1996), one might expect difficulties adhering to the more demanding rules of serial recall, or perseverance of an inappropriate strategy. On the other hand, older adults' difficulty maintaining temporal associations between items may force them to rely on intact semantic associations as the only source of compensation for their reduced temporal coding capability.

Across task and age, slower presentation rates allowed for better recall performance. Although our rate effects were not as pronounced as those in some other studies (e.g., Jackson \& Schneider, 1985), the smaller differences in performance may be attributable to our choice of more conservative rates $(800,1,200$, and 2,400 $\mathrm{msec})$ versus studies using up to 10-sec ISIs (e.g., Jackson \& Schneider, 1985). Of particular interest, however, was whether age differences in associative contiguity might be moderated by rate. We did find a marginally significant three-way interaction of rate, age, and task, suggesting that during serial recall, older adults may benefit more (in terms of making accurate temporal recall transitions) from the longer presentation times than do young adults. However, we did not find a similar interaction for the correlations between lag +1 transitions and overall recall accuracy. Thus, although added time between presented words might have allowed the older participants to begin to form and benefit from temporal associations between list items, we did not find conclusive evidence for this with the modest rates tested here.

Taken together, our results are consistent with theories that predict age-related declines in temporal associations in episodic memory tasks. Older adults are impaired in their ability to make effective use of temporal context, although this deficit may be alleviated with slower presentation rates. In addition, in relation to young adults (who relied less on semantic associations during the temporal context-dependent serial recall task), older adults were less flexible in their persistent use of semantic information. These effects combine to differentially affect older adults' performance on serial recall tasks and open the 
possibility that age-related increases in reliance on semantic memory - even when such reliance conflicts with task goals - may be apparent in other types of memory tasks.

\section{AUTHOR NOTE}

J.D.G. is now at Yale University, J.E.P. is now at the University of Pennsylvania, and K.M.A. is now at Indiana University. The authors acknowledge support from NIH Grants AG15852 and AG04517 to A.W. and MH55687 to M.J.K. We also gratefully acknowledge support from the W. M. Keck Foundation. We thank Simon Dennis for assistance in obtaining LSA values. Correspondence concerning this article should be addressed to A. Wingfield, Volen National Center for Complex Systems, MS 013, Brandeis University, Waltham, MA 02454-9110 (e-mail: wingfield@brandeis.edu).

\section{REFERENCES}

Addis, K. M., \& Kahana, M. J. (2004). Decomposing serial learning: What is missing from the learning curve? Psychonomic Bulletin \& Review, 11, 118-124.

Bä́ckman, L., Small, B. J., \& Wahlin, Å. (2001). Aging and memory: Cognitive and biological perspectives. In J. E. Birren \& K. W. Schaie (Eds.), Handbook of the psychology of aging (5th ed., pp. 349-377). San Diego: Academic Press.

BousfieLD, W. A. (1953). The occurrence of clustering in the recall of randomly arranged associates. Journal of General Psychology, 49, 229-240.

BurKe, D. M., \& Light, L. L. (1981). Memory and aging: The role of retrieval processes. Psychological Bulletin, 90, 513-546.

BurKe, D. M., \& MACKAY, D. G. (1997). Memory, language, and ageing. Philosophical Transactions of the Royal Society B, 352, 1845-1856.

Cabeza, R., Anderson, N. D., Houle, S., Mangels, J. A., \& NyBERG, L. (2000). Age-related differences in neural activity during item and temporal-order memory retrieval: A positron emission tomography study. Journal of Cognitive Neuroscience, 12, 197-206.

CraIK, F. I. M. (1968). Two components in free recall. Journal of Verbal Learning \& Verbal Behavior, 7, 996-1004.

Craik, F. I. M. (1977). Age differences in human memory. In J. E. Birren \& K. W. Schaie (Eds.), Handbook of the psychology of aging (pp. 384-420). New York: Van Nostrand Reinhold.

DEESE, J. (1957). Serial organization in the recall of disconnected items. Psychological Reports, 3, 577-582.

Deese, J., \& Kaufman, R. A. (1957). Serial effects in recall of unorganized and sequentially organized verbal material. Journal of Experimental Psychology, 54, 180-187.

DrewnowsKi, A., \& Murdock, B. B., JR. (1980). The role of auditory features in memory span for words. Journal of Experimental Psychology: Human Learning \& Memory, 6, 319-332.

Glanzer, M., \& CunitZ, A. R. (1966). Two storage mechanisms in free recall. Journal of Verbal Learning \& Verbal Behavior, 5, 351-360.

Howard, M. W., \& Kahana, M. J. (1999). Contextual variability and serial position effects in free recall. Journal of Experimental Psychology: Learning, Memory, \& Cognition, 25, 923-941.

Howard, M. W., \& KaHANA, M. J. (2002a). A distributed representation of temporal context. Journal of Mathematical Psychology, 46, 269-299.

Howard, M. W., \& KaHANA, M. J. (2002b). When does semantic similarity help episodic retrieval? Journal of Memory \& Language, 46, 85-98.

Howard, M. W., Kahana, M. J., \& Wingfield, A. (2006). Aging and contextual binding: Modeling recency and lag recency effects with the temporal context model. Psychonomic Bulletin \& Review, 13, 439-445.

JACKSON, D. K., \& SchneIDER, H. G. (1985). Age, organization, and memory: Effects of presentation rate and rehearsal strategy. Psychological Reports, 56, 471-479.

JAHNKe, J. C. (1965). Primacy and recency effects in serial-position curves of immediate recall. Journal of Experimental Psychology, 70, 130-132.

KahanA, M. J. (1996). Associative retrieval processes in free recall. Memory \& Cognition, 24, 103-109.

Kahana, M. J., Howard, M. W., Zaromb, F., \& Wingfield, A. (2002). Age dissociates recency and lag recency effects in free recall. Journal of Experimental Psychology: Learning, Memory, \& Cognition, 28, 530-540.

KAUSLER, D. H. (1994). Learning and memory in normal aging. San Diego: Academic Press.

Klein, K. A., Addis, K. M., \& Kahana, M. J. (2005). A comparative analysis of serial and free recall. Memory \& Cognition, 33, 833-839.

KUČERA, H., \& FRANCIS, W. N. (1967). Computational analysis of presentday American English. Providence, RI: Brown University Press.

LAMING, D. (1999). Testing the idea of distinct storage mechanisms in memory. International Journal of Psychology, 34, 419-426.

LANDAUER, T. K., \& DumaIs, S. T. (1997). A solution to Plato's problem: The latent semantic analysis theory of acquisition, induction, and representation of knowledge. Psychological Review, 104, 211-240.

Lewandowsky, S., Brown, G. D. A., Wright, T., \& Nimmo, L. M. (2006). Timeless memory: Evidence against temporal distinctiveness models of short-term memory for serial order. Journal of Memory \& Language, 54, 20-38.

Light, L. L. (1991). Memory and aging: Four hypotheses in search of data. Annual Review of Psychology, 42, 333-376.

Murdock, B. B., JR. (1962). The serial position effect of free recall. Journal of Experimental Psychology, 64, 482-488.

NAveh-Benjamin, M. (2000). Adult age differences in memory performance: Tests of an associative deficit hypothesis. Journal of Experimental Psychology: Learning, Memory, \& Cognition, 26, 1170-1187.

Naveh-Benjamin, M., Guez, J., \& Shulman, S. (2004). Older adults' associative deficit in episodic memory: Assessing the role of decline in attentional resources. Psychonomic Bulletin \& Review, 11, 1067-1073.

Naveh-Benjamin, M., Hussain, Z., Guez, J., \& Bar-On, M. (2003). Adult age differences in episodic memory: Further support for an associative-deficit hypothesis. Journal of Experimental Psychology: Learning, Memory, \& Cognition, 29, 826-837.

Nyberg, L., Sandblom, J., Jones, S., Neely, A. S., Petersson, K. M., INGVAR, M., \& BÄCKMAN, L. (2003). Neural correlates of trainingrelated memory improvement in adulthood and aging. Proceedings of the National Academy of Sciences, 100, 13728-13733.

Reeves, A., \& Sperling, G. (1986). Attention gating in short-term visual memory. Psychological Review, 93, 180-206.

Salthouse, T. A. (1991). Theoretical perspectives on cognitive aging. Hillsdale, NJ: Erlbaum.

SAlthouse, T. A. (1996). The processing-speed theory of adult age differences in cognition. Psychological Review, 103, 403-428.

Tulving, E. (1983). Elements of episodic memory. Oxford: Oxford University Press, Clarendon Press.

WeCHSLER, D. (1997). Wechsler Adult Intelligence Scale (3rd ed.). New York: Psychological Corporation.

WEST, R. L. (1996). An application of prefrontal cortex function theory to cognitive aging. Psychological Bulletin, 120, 272-292.

Wingfield, A., \& Kahana, M. J. (2002). The dynamics of memory retrieval in older adulthood. Canadian Journal of Experimental Psychology, 56, 187-199.

WingField, A., LindField, K. C., \& Kahana, M. J. (1998). Adult age differences in the temporal characteristics of category free recall. Psychology \& Aging, 13, 256-266.

Zacks, R. T., Hasher, L., \& LI, K. Z. H. (2000). Human memory. In F. I. M. Craik \& T. A. Salthouse (Eds.), The handbook of aging and cognition (2nd ed., pp. 293-357). Mahwah, NJ: Erlbaum.

(Manuscript received August 16, 2007; revision accepted for publication February 18, 2008.) 